Gazi University
Journal of Science
http://dergipark.gov.tr/gujs

\title{
Application of Grey Wolf Optimization Algorithm: Recent Trends, Issues, and Possible Horizons
}

\author{
Emmanuel Gbenga DADA ${ }^{1, *}$ (D), Stephen Bassi JOSEPH ${ }^{(1)}$, David Opeoluwa OYEWOLA ${ }^{3}$, Alaba \\ Ayotunde FADELE ${ }^{4}$ (i) , Haruna CHIROMA ${ }^{5}$ (i) , Shafi'i Muhammad ABDULHAMID ${ }^{6}$ \\ ${ }^{1}$ Department of Mathematical Sciences, University of Maiduguri, Maiduguri, Nigeria \\ ${ }^{2}$ Department of Computer Engineering, University of Maiduguri, Maiduguri, Nigeria \\ ${ }^{3}$ Department of Mathematics and Computer Science, Federal University Kashere, Gombe, Nigeria \\ ${ }^{4}$ Department of Computer Science, Federal College of Education, Zaria, Nigeria \\ ${ }^{5}$ University of Hafr Al Batin, College of Computer Science and Engineering, Hafr Al Batin, Saudi Arabia \\ ${ }^{6}$ Department of Cyber Security Science, Federal University of Technology Minna, Minna, Nigeria
}

\author{
Highlights \\ - Comprehensive study on the recent trends and progress of GWO algorithm. \\ - Elaborate study of several GWO variants available in literature. \\ - Limitations of existing variants of GWO algorithms. \\ - Applications and possible horizon of GWO algorithms. \\ - Case studies of GWO algorithms in literature.
}

\section{Article Info}

Received: 04 Nov 2020 Accepted: 11 May 2021

\section{Keywords}

Grey wolf optimisation Single objective gwo Multi-objective gwo Hybrid gwo

\begin{abstract}
The development of Grey Wolf Optimisation (GWO) Algorithm was motivated by the biological behaviours of swarm of wolves hunting for prey. This paper presents recent progress on Grey Wolf Optimization (GWO) algorithm, its variants and their applications, issues, and likely prospects. The review revealed that opportunities still exists for development of more robust and stable variants of GWO that will overcome the shortcomings of existing variants. This review has the potential to stimulate researchers in the area of nature-inspired algorithms to further advance the effectiveness of the GWO and its ability to solve problems. Such problems can be real-life, complicated and nonlinear optimization problems in different domain of human endeavour. Suggestions for new research directions that have the capacity to increase the performance of GWO are presented. It is expected that this paper will serve as reading material for beginners whereas experienced researchers can also use it as an article yardstick for further development of GWO algorithms.
\end{abstract}

\section{INTRODUCTION}

Swarm Intelligence (SI) has inspired many researchers to develop optimization algorithms that mimic animals' behaviour. SI draws is patterned after the activities of living creatures like bat, fish, cattle, insects, birds, and ants [1]. Several bio-inspired algorithms are already existing in the literatures which include particle swarm optimization (PSO) [2], artificial bee colony (ABC) [3], Bat algorithm [4], Bees algorithms [5], Monkey Search [6], Glow warm swarm [7], bacterial foraging [8], Fish-swarm algorithm [9], Cat swarm [10] and others. Fister et al. in [11] presented a concise review of bio-inspired algorithms highlighting the different types of bio-inspired algorithms in literature. Lately, Flower Pollination Algorithm (FPA) [12], Chicken Swarm Optimization (CSO) [13], Approximate Muscle Guided Beam Search (AMGBS) [14], Magnetotactic Bacteria Optimization based on Moment Migration (MBOMM) [15], Elephant Herding Optimization [16], Bull optimization algorithm [17] and modified imperialist competitive algorithm with invasive weed optimization (MICA-IWO) were proposed. Depicted in Figure 1 are the different SI-based algorithms available in literature. 
Lately, GWO algorithm is among the newly proposed SI-based optimization algorithms. GWO drew its inspiration from the behaviour of a swarm of wolves [18]. The original GWO algorithm was initially proposed by Mirjalili et al. [18]. Since its development, several researchers have applied it to solve different problems. Gholizadeh [19] used GWO to proffer solution to an optimization problem of grids having many layers bearing in mind their nonlinear behaviour. Experimental results showed that GWO outperforms the other methods in locating the best design of nonlinear double-layer grids. Mirjalili [20] applied the GWO to train multi-layer perceptron (MLP). The authors did a comparison between the results of GWO and that of PSO, GA, ACO and EA. Saremi et al. [21] fused GWO with the evolutionary population dynamic (EPD) to enhance the performance of the traditional GWO approach. Experimental outcomes revealed that the new hybrid algorithm performs better than GWO.

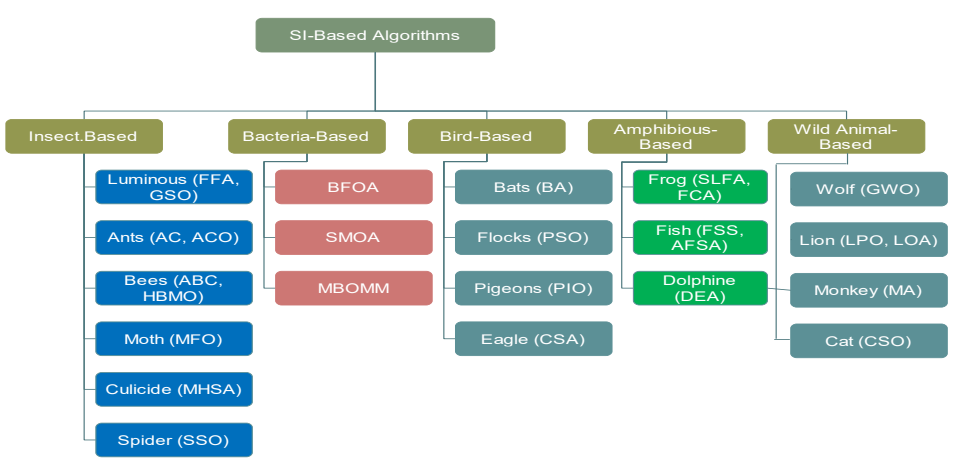

Figure 1. SI-based Algorithms

Sulaiman et al. [22] employed GWO to find solution to optimal reactive power dispatch (ORPD) problem. It has also been used for power flow optimisation [23], control of DC motor [24], power system load frequency control [25] and others. Figure 2 is a pictorial representation showing GWO variants and their area of applications.

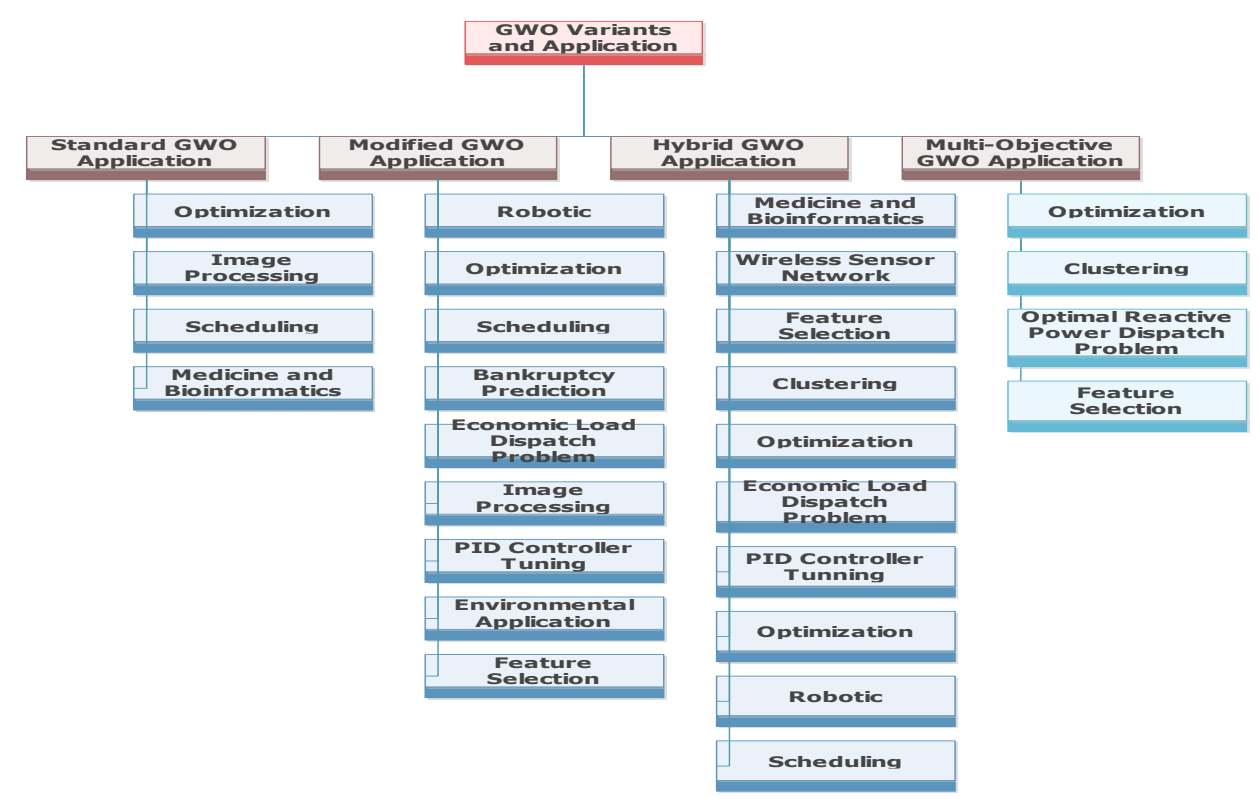

Figure 2. GWO Variants and their Applications

GWO has drawn substantial attention from the researchers and has been widely employed to find solution to everyday optimization issues like parameter estimation in surface waves. GWO has become the choice bio-inspired algorithm for solving several problems because of its ease of implementation and fewer tuning parameters that are needed to make it attain the global optimal [26]. Among the most newly developed bioinspired algorithms, GWO has been one of the most popular and has been applied in many fields as contrasted to CSO, MGBS, and MBOMM. This paper hereby analyse the uses of the GWO in solving real- 
life problems some of which are complicated, dynamic and nonlinear in nature. The novelty of this work are summarised below:

a. Comprehensive study on the recent trends and progress of GWO algorithm, their variants and application was done.

b. Applications of GWO and their variants were identified and discussed. The applications areas such as optimization, robotic, PID controller tuning, environmental application, economic load dispatch problem, feature selection, clustering, scheduling, wireless sensor network, image processing and medicine and bioinformatics were extensively discussed.

c. An elaborate study of several GWO variants available in literature over the period (2015-2020) was presented in this paper.

d. Identification of limitations of existing variants of grey wolf optimization algorithms to highlight the issues and possible horizons.

This review will aid researchers to quickly understand the applications of GWO algorithm. And also discover areas that need further research and develop new variants of GWO that can solve problems in other fields that are yet to be explored. The remainder of this work are as follows: section 2 gives explanation on the GWO algorithm. Section 3 discusses the areas where GWO has been applied and the outcomes of the papers. In section 4, presents the issues and new horizons that researchers can work on to make GWO algorithms better. Section 5 is the concluding remarks, and further suggestions for future research on the GWO algorithms are discussed in this paper.

\section{PREVIOUS REVIEWS}

In recent years, GWO has caught the attention of the research community worldwide. In this section, we present related reviews done earlier on GWO chronologically according to the year of review. Faris et al [27] in their work titled "Grey Wolf Optimizer: a review of recent variants and applications" chronicled the details of GWO, their variants and applications. Many research papers based on GWO were overviewed and outlined. GWO framework, operations, and the theoretical foundation were extensively discussed. Hatta et al [28] did a study of the trend, principles and how GWO has been applied from 2014 to 2017. The authors studied several publications that are interrelated to GWO. Their study examined the applications of GWO to different areas.

Panda and Das [29] presented a brief survey on GWO and its applications. The paper described GWO algorithm, its mathematical model and pseudocode. Twelve different applications of GWO was also discussed in their work. The authors, however, did not discuss the variants of GWO that are already in existence. However, time of coverage was not presented. Al-Tashi et al [30] did a review on GWO for solving feature selection methods for classification problem. The paper discussed general framework of feature selection. The authors took their time to discuss the different GWO-based feature selection techniques and their application in different fields. However, the review is limited to only feature selection using GWO. Also, the review did not discuss extensively the variants of GWO. Also, it was only the summary of the areas of application that was presented. Negi et al [31] reviewed the advances in the development of GWO algorithms and how they are being used to solving problem in various areas of human endeavours.

Table 1. Summary of previous reviews in GWO Algorithm

\begin{tabular}{|c|c|c|c|c|c|c|c|}
\hline Previous Reviews & 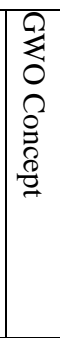 & 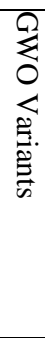 & $\begin{array}{l}\text { D. } \\
0 \\
0.0 \\
0 \\
0 \\
0 \\
0 \\
0 \\
0 \\
0 \\
2 \\
0\end{array}$ & 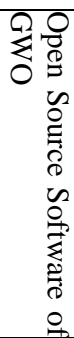 & 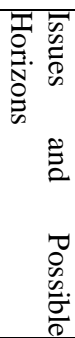 & 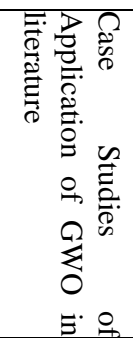 & 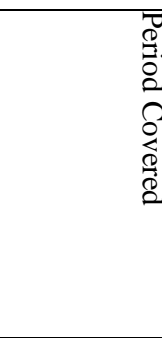 \\
\hline Faris et al [27] & $\sqrt{ }$ & $\sqrt{ }$ & $\sqrt{ }$ & $\sqrt{ }$ & & & 2014-2017 \\
\hline
\end{tabular}




\begin{tabular}{|l|l|l|l|l|l|l|l|}
\hline Hatta et al [28] & $\sqrt{ }$ & $\sqrt{ }$ & $\sqrt{ }$ & & & & 2014-2018 \\
\hline Panda and Das [29] & $\sqrt{ }$ & $\sqrt{ }$ & $\sqrt{ }$ & & & & 2014-2019 \\
\hline Al-Tashi et al [30] & $\sqrt{ }$ & $\sqrt{ }$ & $\sqrt{ }$ & & & & 2014-2020 \\
\hline Negi et al [31] & $\sqrt{ }$ & $\sqrt{ }$ & $\sqrt{ }$ & & & & $2014-2020$ \\
\hline & $\sqrt{ }$ & $\sqrt{ }$ & $\sqrt{ }$ & & $\sqrt{ }$ & $\sqrt{ }$ & $2014-2020$ \\
\hline
\end{tabular}

This review is not the same as the earlier ones presented in the above paragraphs. The review explains the workings of the GWO algorithms, the different variants of GWO, their applications, case studies from literature, and open challenges. This review covers all recent research articles on GWO (2015 to 2020) that are considered useful for development of more efficient and robust variants of GWO. The summation of previous reviews are in Table 1 below.

\section{GREY WOLF OPTIMISATION (GWO) ALGORITHM}

This section describes the GWO algorithm. GWO imitates the social hierarchy and the clever hunting displayed by a swarm of grey wolves. Naturally, grey wolves live in a group of between 5 to 12 individuals. Grey wolves sternly live in a social hierarchy. As depicted in Figure 3, the leaders of a group of grey wolves known as "alpha" are male and female wolves that are in charge of decision making on behalf of other wolves in the group. Such decisions include where to sleep, time to awake and hunting for preys. Generally, other wolves in the group must comply with the decision made by alpha. Nevertheless, in some cases, some classless actions in the social hierarchy of grey wolves are witnessed. In such cases, alpha can obey other wolves in the group. In meetings, other wolves sanction the decision made by alpha by lowering down their tails. It is worth noting that it is not compulsory that alpha should be the strongest wolf in the group. The chief responsibility of alpha is to oversee the group.

The most important characteristics of a group of grey wolves are their self-restraint and orderliness. After alpha, the next echelon in the social hierarchy of grey wolves is beta and the responsibility of beta is to assist alpha in decision-making processes. Any of the male or female wolves can be beta and beta can be the most suitable entrant to replace alpha at any time when old age catch up with any of them or any one of them is deceased. It is demanded that beta reverence and obey alpha, but he/she can control other wolves in his/her hierarchy. Beta serves as a counsellor for alpha and is in charge of punishing any erring individual in the group. The beta emphasizes the commands of alpha and provides responses of group members to alpha.

The lowest level in a group of grey wolves is an omega that acts like the victim. It is compulsory for the wolves at this level to submit themselves to the commands of other wolves of higher hierarchy and they are not permitted to eat food until other wolves in other groups have eaten. Though omega looks like the most trivial wolves in the group, without an omega it will be difficult to detect the existence of internal conflict and other problems. The reason for this is that omega is saddled with the responsibility of exposing the existence of cruelty in the group and the displeasure of other wolves. These make other wolves to be contented and also preserve the central organization of grey wolves. Occasionally, omega functions as child-minder in the group.

The rest of the wolves, apart from alpha, beta, and omega, are termed secondary (delta). The delta wolves submit to the alpha and beta wolves and rule over the omega wolves. They function as spies, watchmen, elders, hunters, and guards in the group. Spies are in charge of taking care of borderlines and area. They also raise warning alarm of any hazard faced by the group. Watchmen are responsible for safekeeping the group. The elders are veteran wolves that are qualified to be alpha and beta. Hunters assist alpha and beta in pursuing preys and getting food ready for the group, while guards take care of the feeble, sick, and injured wolves [1]. It is worthy to note that dominance decreases from top of the hierarchy downwards. Figure 3 summarises the social hierarchy of grey wolves and the roles each hierarchy plays in the group. 


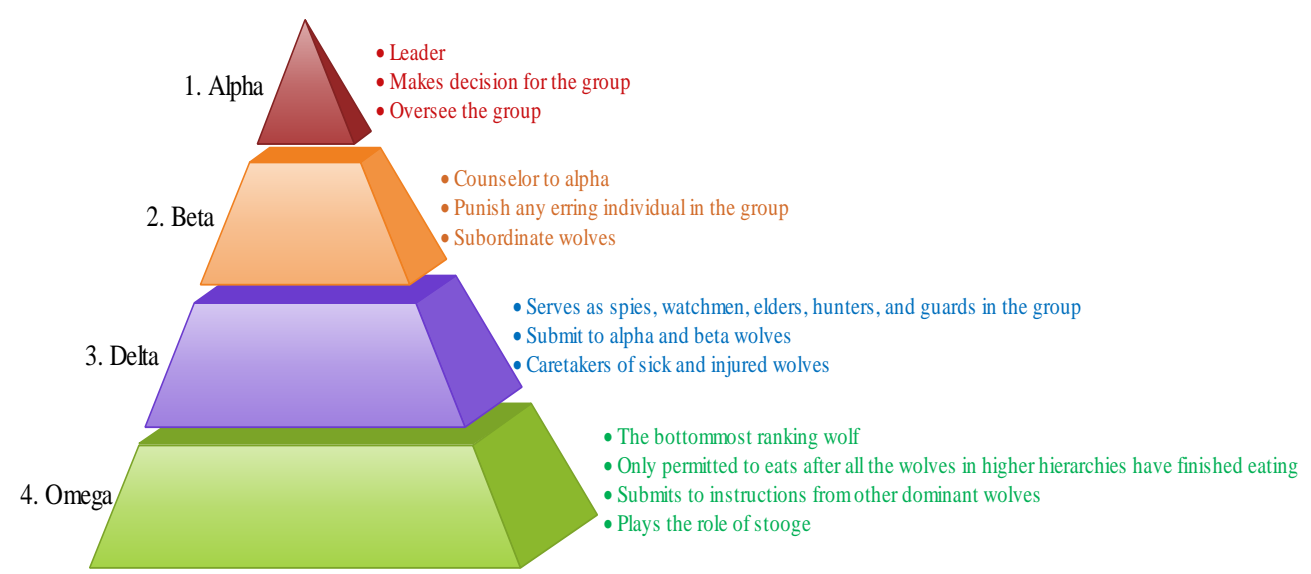

Figure 3. Distribution of Grey Wolves Social Hierarchy and their responsibilities

Besides the social hierarchy that exists in a group of grey wolves, collective hunting is another fascinating communal behaviours of grey wolves. The grey wolves' hunting includes the steps represented in Figure 4:

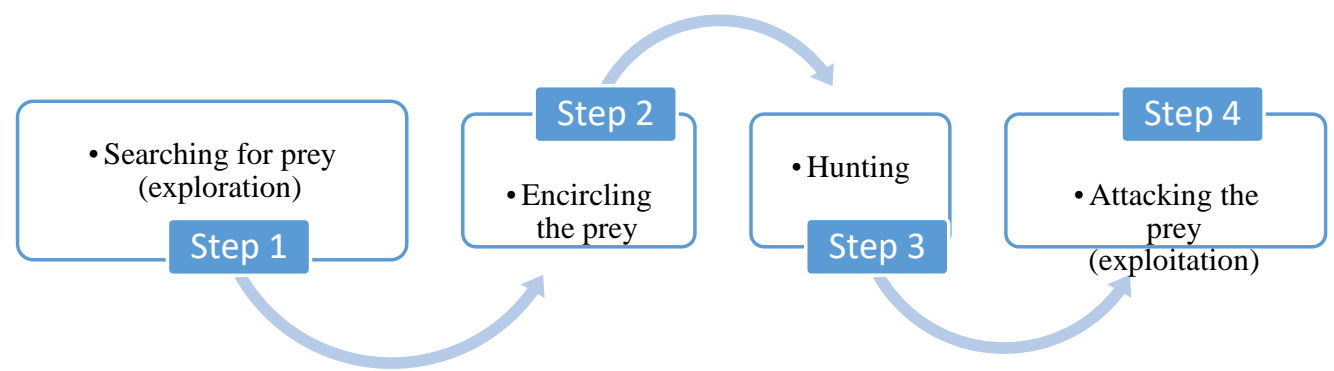

Figure 4. Grey Wolf Hunting process

The GWO algorithm modeled communal activities of grey wolves' group which are social hierarchy and hunting method.

\subsection{Social Hierarchy}

In the social hierarchy of grey wolves in the GWO algorithm, the best solution is represented as alpha $\alpha$. Consequently, the second and third best solutions are represented as beta $\beta$ and delta $\delta$ respectively, and another solution is taken as omega $\omega$. In the GWO algorithm, hunting (optimization) is guided by $\alpha, \beta$ and $\delta$ wolves while $\omega$ go behind them.

\subsection{Searching for the Prey (Exploration)}

Grey wolves usually comb the environment for prey based on the positions of $\alpha, \beta$, and $\delta$. They wander away from each other to search for the location of prey and then congregate to attack the prey. Let us assume that $\vec{A}$ is a random vector that is between the range of -1 and 1 to compel the search agent to wander from the prey, which underlines the global search in GWO. When $|\mathrm{A}|$ is greater than 1 the grey wolf is compelled to deviate from the prey (local optimum) to look for superior results in the decision space.

This algorithm possesses an additional element $(\vec{C})$ that aids the algorithm to arrive at new solutions. According to Equation (4), the elements of a vector $\vec{C}$ are in the range of interval $[0,2]$. The vector $\vec{C}$ supplies non-linear weights to the prey to arbitrarily accentuate $(c>1)$ or trivialise $(c<1)$ the influence of the prey in circumscribing the distance according to Equation (1). The GWO algorithm is able to explore more search space randomly through this singular factor. It also allows the search agent to escape been caught in the local optima during the process of optimization. Contrary to what we have in A, the decrement in $\mathrm{C}$ is nonlinear. The $\mathrm{C}$ vector is needed from the beginning to the end of the iteration process to enhance 
global search in the decision space as it prevents the search from been unable to move further in the local optima. $\mathrm{C}$ is employed as a barricade witnessed in the natural hunting process of grey wolves. This method of seeking out prey hinders grey wolves from swiftly advancing towards the prey. This is exactly the function of $\mathrm{C}$ in GWO algorithm all through the optimization operation.

\subsection{Encircling the Prey}

Based on the abovementioned, grey wolves during their hunting activity encompass their target. The grey wolves' action of surrounding their prey is stated as below [18]:

$\vec{D}=\left|\vec{C} \cdot \vec{X}_{p}(t)-\vec{X}(t)\right|$

$\overrightarrow{X(t+1)}=\vec{X}_{p}(t)-\vec{A} \cdot \vec{D}$

Assuming t is iteration number; $\vec{A}$ and $\vec{C}$ are coefficient vectors; $\vec{X}_{p}$ is a vector of the prey's positions; $\vec{X}$ is a vector of the grey wolf's positions; and $\vec{D}$ is computed vector employed to denote a new position of the grey wolf. $\vec{A}$ and $\vec{C}$ can be computed using the formulas below:

$\vec{A}=2 \vec{a} \cdot \vec{r}_{1}-\vec{a}$

$\vec{C}=2 \cdot \vec{r}_{2}$.

Assuming $\vec{A}$ is vector whose value is to reduce linearly from 2 to 0 over the iterations; and $\vec{r}_{1}$ and $\vec{r}_{2}$ are random vectors in $[0,1]$. As depicted in Figure 1, the position of a grey wolf at $(\mathrm{x}, \mathrm{y})$ can change depending on the position of prey at (x', y'). Various locations to the most ideal agent can be attained with regard to the present position by controlling $\vec{A}$ and $\vec{C}$, such as, by setting $\vec{A}$ to $[1,0]$ and $C$ to $[1,1]$. The new position of the grey wolf is now ( $\left.\mathrm{x}^{\prime}-\mathrm{x}, \mathrm{y}^{\prime}\right)$.

The random vectors $\vec{r}_{1}$ and $\vec{r}_{2}$ allow the grey wolf to choose any position or node. Hence, a grey wolf can be positioned in any arbitrary position close to the prey. The position is computed using Equations (1) and (2). In a similar manner, grey wolves can change their position to any node of a hypercube in an ndimensional decision space close to the optimal solution (position of the prey). They possess the capacity to differentiate the location of prey apart from others and encircle it. Generally, the hunting process is directed by $\alpha$ and $\beta$, while $\delta$ offer help for $\alpha$. Hence, to mimic the stalking behaviour of grey wolves, it is presumed that $\alpha$ (most viable candidate for the solution), $\beta$ and $\delta$ are more cognizant of the likely bearings of the prey. Consequently, GWO retains three most ideal solutions attained to this point and obliges the omega wolves to bring up to date their positions to attain the ideal place in the decision space. According to [14] such a hunting behavior can be modelled in an optimization algorithm by expressing it as:

$\vec{D}_{\alpha}=\left|\vec{C}_{1} \cdot X_{a}-\vec{X}\right| \vec{D}_{\beta}=\left|\vec{C}_{2} \cdot X_{\beta}-\vec{X}\right| \vec{D}_{\delta}=\left|\vec{C}_{3} \cdot X_{\delta}-\vec{X}\right|$

$\left.\left.\vec{X}_{1}=\vec{X}_{\alpha}-A_{j} \cdot\left(\vec{D}_{\alpha}\right), \vec{X}_{2}=\vec{X}_{\beta}-A_{2} \cdot \overrightarrow{(D}_{\beta}\right), \vec{X}_{3}=\vec{X}_{\delta}-A_{2} \cdot \overrightarrow{(D}_{\delta}\right)$

$\vec{X}(t+1)=\frac{\overline{x_{1}+x_{2}+x_{3}}}{3}$.

Figure 5 depicts the way search agent makes the positions of $\alpha, \beta$, and $\delta$ to be up to date in a two dimensional state space. Based on what we have in Figure 5, the ultimate position (solution) is in a sphere which is stipulated depending on the positions of $\alpha, \beta$, and $\delta$ in the state space. Put differently, $\alpha, \beta$, and $\delta$ evaluate the positions of prey and the remaining wolves and afterward make their new up to date positions arbitrarily close to the prey.

\subsection{Attacking the Prey}

Based on the earlier discussion, grey wolves complete their stalking process by pouncing on the prey until it dies. To imitate the attacking process, the value of $\vec{a}$ is reduced in various iterations. Observe that as $\vec{a}$ 
reduces, the degree of variation of $\vec{a}$ also reduces. Alternatively stated, $\vec{a}$ is a variant in the interval [2a; $2 \mathrm{a}]$. The value decreases from 2 to 0 as the iterations continues, and can be expressed as follows:

$\vec{a}=2-t \cdot \frac{2}{\max _{i} t e r}$

where $\max _{i}$ ter is the sum of iterations done during the optimization and $t$ is the iteration number.

As soon as the stochastic value of $\vec{A}$ is in the interval $[1 ; 1]$. The subsequent position of a wolf can be between the present position of the wolf and the prey position. Figure 5 depicts that when $|\mathrm{A}|<1$ grey wolves will show aggression toward the prey.

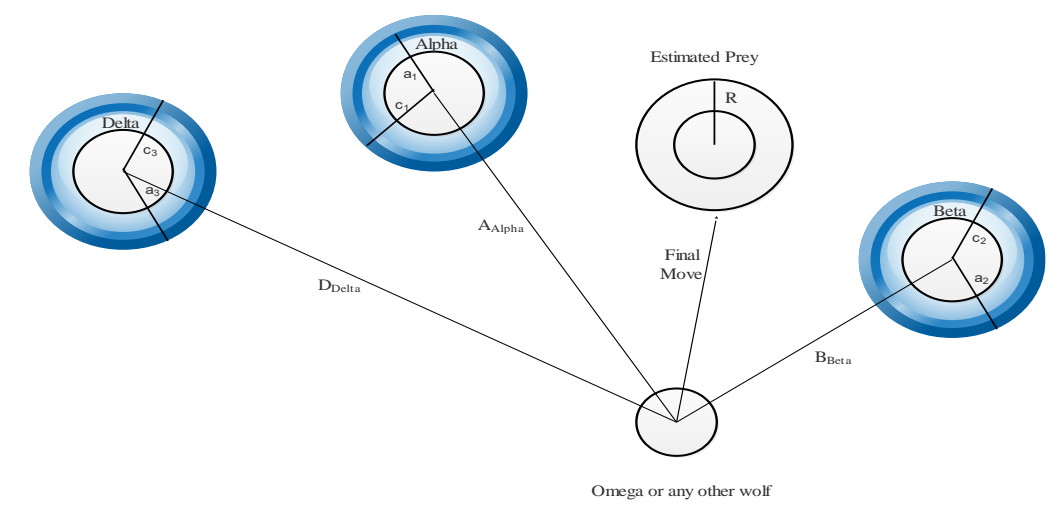

Figure 5. Updating position in GWO algorithm

Through operators made available, the GWO algorithm allows the search agent to make its position up to date using the positions of $\alpha, \beta$, and $\delta$ (approach the animal that is hunted). Nevertheless, GWO also possess some other operators that can be used to compute new solutions. Figures 6 (a) and (b) show how grey wolves explore their environment and find the right prey before encircling and attacking the estimated prey.

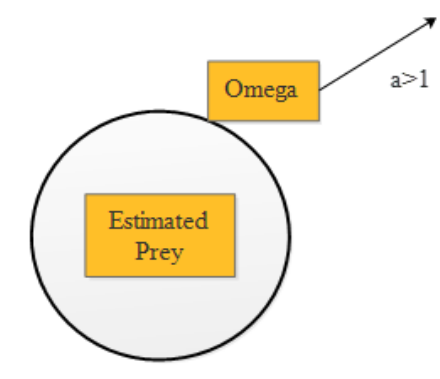

Figure 6(a). Diverging away from a prey to find the right prey

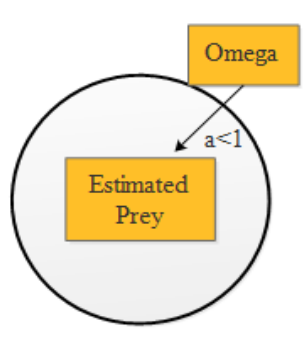

Figure 6(b). Finding a suitable location to encircle and attack the prey

\section{APPLICATIONS OF GREY WOLF OPTIMIZATION ALGORITHMS}

\subsection{Engineering Design Problems}

Optimization of engineering operations is a very important aspect of engineering. It has direct bearing on the living standard of people. Grey Wolf Optimization, due to its flexibility has been adapted to solve many engineering problem. These applications are discussed extensively in this section.

\section{Design and controllers tuning}

Mirjalili, Mirjalili and Lewis [18] developed Grey Wolf Optimization Algorithm through inspiration from hunting behaviour of a swarm of wolves. The performance of the algorithm was then evaluated, and the 
results are substantiated by a relative study with some popular algorithms [18]. Findings indicated that GWO produced excellent results compared to celebrated heuristics algorithms compared in the study. The authors in [16] also used the algorithm to proffer solution to some engineering design problems and discussed how the proposed technique can be applied to the design of optical instruments.

However, like other bio-inspired optimization algorithms, GWO has its shortcomings. For example, the algorithm is regularly stuck in the local optimal when handling high-dimensional nonlinear functions. Moreover, GWO does not converge easily in some cases. This is because GWO finds it difficult to maintain a balance between its exploitation and exploration process. Due to these drawbacks, researchers are working on developing high-performance variants of GWO to surmount these weaknesses. Yang et al. [32] used grouped grey wolf optimizer (GGWO) to find the optimal parameters of synergistic PID controllers of doubly-fed induction generator based wind turbine. GGWO was evaluated against GA, PSO, Moth Flame Optimisation and GWO. Lal, Barisal and Tripathy [33] applied GWO based Fuzzy PID controller for optimal gains of the fuzzy-based PID controllers in interconnected Hydro-thermal power system for automatic generation control (AGC). The proposed system outperformed DE and PSO. Precup et al. [34] used a new technique that uses GWO algorithm to tune the parameters of Takagi-Sugeno PI-FCs for a class of nonlinear servo systems. Performance of the proposed system was better than GWO, PSO and GSA. The combination of differential evolution, GWO and fuzzy PID controller was applied by Debnath, Mallick and Sahu [35] to automatically control multi-source interconnected power system.

\section{Scheduling}

Lu et al. in [36] proposed a multi-objective discrete grey wolf optimizer (MODGWO) for a solving practical scheduling problem in welding production. To evaluate the effectiveness of the proposed MODGWO, they compare it with NSGA-II and SPEA2 on a set of instances. Results demonstrated that the MODGWO outperforms NSGA-II and SPEA2. Panwara et al. [37] used Binary Grey Wolf Optimizer (BGWO) to decide the dedication timetable of UC problem. The outcomes of their experiments show the superiority of BGWO in proffering solution to UC problem for lesser, intermediate and extensive systems in comparison to other approaches considered in the study. The authors in [38] used GWO to provide solution to assembly flow shop scheduling problem. Findings show that GWO outperform PSO and CSA. Kamboj [39] used the hybrid of PSO and GWO to proffer solution to the problem of finding the minimum cost dispatch of accessible generation resources to satisfy predicted electric power demand for a specific period of time. The combination and Ant colony optimisation (ACO) and GWO was used by Rashid [40] to solve assembly system scheduling problem.

\section{Robotic and path planning}

Zhang et al. [41] used GWO to solve Unmanned Combat Aerial Vehicle (UCAV) two-dimension path planning problem. The performance of GWO proved to be very competitive when compared with other techniques. The performance of GWO was as good as that of other advanced algorithms compared. Jain et al [42] develop a hybrid system comprising of PSO and GWO to solve robotic odour localization problem.

\section{Optimal power dispatch problems}

Sulaiman et al. [22] used GWO to provide solution to optimal reactive power dispatch (ORPD) problem and compared its performance with other methods. The results showed that GWO possesses suitable ideal solution compared to others. Pradhan et al. [43] applied GWO for solving the best operating plan of economic load dispatch (ELD) problem. The algorithm was tested on four test systems. Results demonstrated that the GWO is performs better than GA-API, SDE, TLB, QOTLBO and KHA. Pradhan, Roy and Pal [44] used the Oppositional based grey wolf optimization (OGWO) algorithm for solving optimal operating strategy of economic load dispatch (ELD) problem. The performance of OGWO is superior to that of GWO, OIWO, SDE and ORC-CRO. Jayabarathi et al. [45] applied the Hybrid GWO (HGWO) to provide solution to economic dispatch problems. The results obtained showed that HGWO outperform GWO, PSO, SCA, FAPSO, CSO, KHA and CE-SQP. Chopra, Kumar and Mehta [46] hybridized PSO and GWO and used the new approach to provide solution to convex economic load dispatch 
problem. The combination of PSO, GWO and SVM was used by Eid and Abraham [47] to identify different species of plants.

\section{Image processing}

Vinothini and Bakkiyaraj [48] applied GWO for improving contrast, preserving brightness and quality augmentation of low dynamic range images. The performance of GWO was superior to that of GA as shown from their experiment. Srivathsan Lakshminarayanan [49] used GWO to effectively plan operation of Energy Storage Unit (ESU) in a green smart home to lessen power consumption and to stabilize the load on the network. The result shows that GWO outperforms PSO. Medjaheda et al. [50] used the GWO algorithm to reduce dimensionality of hyperspectral images. The performance of GWO proves to be superior to PSO, GSA, DE, EP and BBA.

\subsection{Machine Learning}

GWO has found application in several machine learning operations [51-56]. The applications are discussed in detail below.

\section{Optimization problem}

Mirjalili et al. [51] used the multi-objective grey wolf optimizer (MOGWO) to solve multi-criterion optimization problem. Experimental results indicated that MOGWO algorithm's performance is good and outperforms MOEA/D and MOPSO. Kohli and Arora [52] applied chaotic GWO algorithm to solve constrained benchmark problems. The effectiveness of CGWO was compared with that of conventional GWO, Firefly Algorithm, FPA and PSO. Singh and Singh [53] applied the hybrid GWO and Sine Cosine Algorithm (SCA) to provide solutions with superior quality for benchmark problems and practical applications in both constrained and unconstrained global optimization problems. The study showed that GWOSCA performed better than other popular algorithms considered in the study. Heidari and Pahlavani [57] used the modified grey wolf optimizer with Lévy flight (LGWO) for providing solution to global and real-life optimization problems. The study concluded that LGWO is significantly better than other optimizers considered in the paper.

Jitkongchuen, Phaidang and Pongtawevirat [58] applied the hybrid of GWO and invasion-based migration operation (IM-GWO) to benchmarking problem. Results showed that IM-GWO have the potential to effectively provide solution to difficult problems that entails locating the best solution among all possible solutions. Singh and Singh [55] applied the fusion of PSO and GWO to enhance the exploratory capacity of GWO and the exploitative ability of PSO. Simulation results shows that the hybridised system outperform both PSO and GWO individually. ElGayyar et al. [56] developed the hybrid of GWO and Bat algorithm to help these algorithms escape from being trapped in the global optimal.

Pan, Dao and Chu [59] proposed the fusion of GWO and FPA for solving optimization problems. Zhang et al. [60] proposed a hybrid system comprising of GWO and Biogeography-based optimization (HBBOG) for finding solution to optimization problems. HBBOG performs better when was compared with that of GWO, DE, PSO, ABC and CS variants. HBBOG proved to have superior performance compared to other algorithms though the performance metrics used are just mean, standard deviation and runtime which in our opinion is not enough to sufficiently prove the supremacy of the proposed algorithm over others. The fusion of GWO and GA was applied by Tawhid and Ali [61] to reduce potential energy function of molecule. The effectiveness of the method was validated using 8 benchmark on different molecule size of up to 200 dimensions.

\section{Clustering}

Jeet [62] applied MOGWGA algorithm to cluster five sample software. Six clustering objectives were considered for the application of these algorithms. The outputs are measured to that of Two-Archive, MOGA and NSGA-II based methods. Results show that MOGWGA algorithm outperforms the two other approaches used for the problem addressed in the paper. Jadhav and Gomathi [63] used the WEGWO to 
decide the best geometric center for the organizing the objects into groups. The proposed algorithm performed better than algorithms compared in the study in term of convergence speed and accuracy.

\section{Bankruptcy prediction}

The issue of bankruptcy in today's financial world cannot be overemphasized. This has led financial institutions to seek for measures to predict and serve warnings to industries and organizations. Wang et al. [64] applied GWO evolving kernel extreme learning machine (GWO-KELM) to bankruptcy prediction. Results of the experiments conducted validate the supremacy of GWO-KELM over six advanced prediction models for bankruptcy. However, GWO-KELM was only tested on a small dataset. Furthermore, feature selection and parameter setting were implemented differently which may reduce prediction accuracy. Hence, as a further research larger datasets can be explored and concurrently applying parameter setting and feature selection to GWO-KELM is open for research.

\section{Feature selection}

Emary et al. [65] applied the Multi-objective GWO (MOGWO) to explore the space of features to locate the best feature subset that both realize data description with little profusion, while maintaining high classification accuracy. The proposed method is assessed against PSO and GA and some optimization problem that deals with locating the most ideal solution for a particular problem. The MOGWO outperform both PSO and GA. Tu, Chen and Liu [66] used multi-strategy ensemble GWO (MEGWO) that integrates three set of search approaches to update the solutions of GWO. Simulation results shows that their proposed technique performed better that other algorithms compared. The downside of their approach is that the classification accuracy on some dataset used for their experiments was very low. Moreover, the technique introduces extra parameters which requires users to train the parameters in order to attain the most ideal setting beforehand. This implies that there is additional computational cost attached to using MEGWO.

$\mathrm{Hu}$, Pan and Chu [67] proposed an improved Binary Grey Wolf Optimizer that can be used for feature selection. The authors used five transfer functions improve the quality of their output. Simulation results using different benchmark functions demonstrated that advanced binary GWO (ABGWO) performs better than conventional BGWO in term of ability to locate best solution, convergence speed, time wastage and minimal classification errors with few features. The performance of improved Binary Grey Wolf algorithm was compared with that of four variants of BGWO and ABGWO. Results illustrated that the Improved BGWO have superior performance compared to other algorithms considered.

\section{Medicine and bioinformatics}

GWO has been used for the classification, storage, and analysis of biochemical and biological information. Li et al. [68] applied a the hybrid of binary GWO and extreme learning machine (ELM) to carry out feature selection for early diagnosis Parkinson's disease diagnosis and breast cancer. Jayapriya and Arock [69] proposed parallel GWO for solving pairwise molecular sequence alignment problem. The authors used a new fitness function that produced optimal corresponded counts for newly created molecular sequences.

Moreover, Mostafa et al. [70] applied the fusion of GWO, statistical image of liver and simple region growing for liver segmentation in computed tomography scan. Simulation results showed that their technique have high classification accuracy. Sahoo and Chandra [71] developed multi-objective grey wolf optimizer to classify cervix lesion. Their approach have the ability to detect precancerous cervical lesion, which is an abnormality in the cells of your cervix that could finally result into cervical cancer. Elhariri [72] used the hybrid of GWO with SVM for Electromyography (EMG) signal classification which has several applications in medicine and bioinformatics. Simulation results demonstrated that the GWO-SVM is as good as that of the other bio-inspired algorithms such as PSO, GA, and ACO.

\section{Wireless sensor networks (WSNs)}

The GWO has also been used to tackle challenges associated with the use of wireless sensor networks (WSNs). Diwan and Khan [73] used the combination of GWO and fuzzy logic for cluster to reduce power 
consumption in network thereby prolonging network life span. GWO algorithm has also been integrated to effectively tackle the problems associated with finding the geometrical position of the sensor node in the network in [28] by identifying the accurate location of unidentified network nodes.

\section{Earth's response to environmental change}

Song et al. [26] used the GWO algorithm for parameter approximation in seismic wave that moves near or parallel to the earth's surface. Their approach was benchmarked on meaningful, meaningless, and field data. The outputs of the GWO algorithm are contrasted to that of GA, the fusion of PSO and Gravitational Search Algorithm (GSA), and gradient-based algorithm. Results from both artificial and authentic data shows that GWO utilized for seismic wave analysis demonstrate a suitable equilibrium between exploration and exploitation that leads in extraordinary escape from local optima and super-convergence concurrently.

\section{PUBLICATIONS TRENDS ON APPLICATIONS OF GREY WOLF OPTIMIZATION ALGORITHM}

Figure 7 shows trend of the publications on the applications of GWO from 2015 to 2020. The publications trend indicated that the GWO is getting attention from academics and the volume of publications is rapidly increasing. The smallest number of published paper on this subject was recorded in 2015 which is expected since the algorithm was proposed in 2014. The highest number of published paper was achieved in 2020 despite the COVID-19 pandemic. This suggests that the volume of papers published on GWO algorithm is rising and is likely to keep on increasing rapidly in future literature.

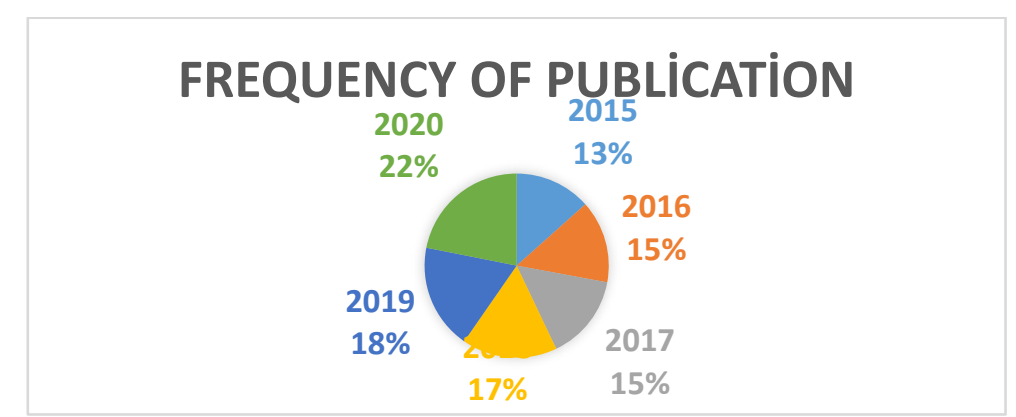

Figure 7. Frequency of Publication of GWO and their applications from 2015-2020

This is an indication that much work still needs to be done to develop more high performing GWO variants. Figure 8 shows the classification of the GWO and the numbers connected to each of the GWO variants exemplify the number of applications found in the literature.

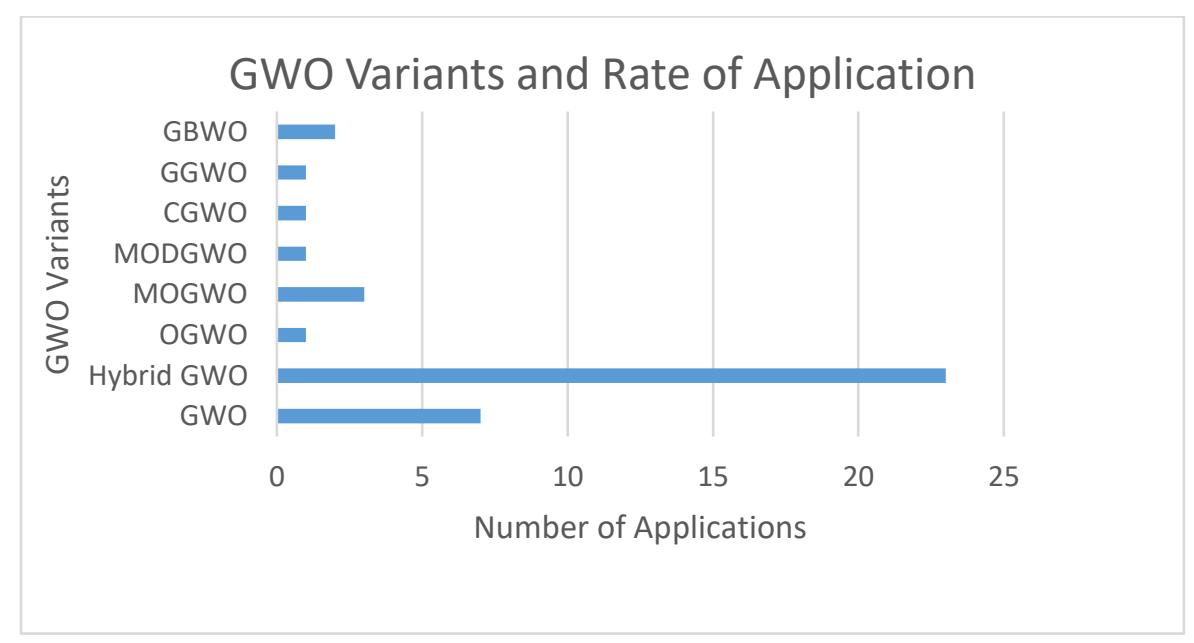

Figure 8. Classification of the variants of GWO and the rate of applications 
From Figure 8, it can be stated that apart from the conventional GWO, the most applied variants of the GWO is the Hybrid GWO (HGWO). Hybridization in GWO is the fusion of GWO with some other metaheuristic algorithms such as. FA, NN, PSO, GA, etc. Hybridization improves the performance of the hybrid algorithm by eliminating the shortcomings of the GWO to enhance the effectiveness and efficiency of the GWO to converge to the optimal solution within a brief duration of time. The integration of two or more optimization algorithms has demonstrated that they possess the capacity to eradicate their inadequacies and take advantage of on their strengths to quickly generate the best solution compared to the individual algorithm [74]. Probably this can be due to its superior performance because of its ability to annex the powers of the different algorithms that made up the hybrid system. This maintains equilibrium between exploration and exploitation thereby improving the quality of the solution generated. The other variants of GWO result from the variations to the tuning parameters of the original GWO.

\section{ISSUES AND POSSIBLE HORIZONS}

The past few years has witnessed a momentous increase in the scope and volume of development of new variants of GWO algorithms and their application to real life problems. However, there are still a number of important open problems even though GWO has been successfully applied to solving many of interesting real-life problems. These problems need to be tackled for GWO to maximize its full potential and to grow into a more reliable conventional part of the computational problem solving toolbox. It was discovered by authors in [75] that GWO has certain defects that are still open for researchers to address. It has been experimentally proven that GWO performs excellently when the best solution for an optimization problem is 0 . On the other hand, when used to solve other problems, its performance deteriorates and becomes obscure or in some circumstances becomes more defective and problematic. They also discovered that even when GWO is used to solve identical optimization function, the performance is directly proportional to how far the function's best solution is from 0 . In other word, the farther the distance of the most ideal solution from 0 , the poorer its performance. This attribute is not good for an optimization algorithm as it places a limitation on GWO and its applications. It cannot therefore be counted as a preferred algorithm for solving problems. It must also be noted that GWO based research are yet to be substantiated [75]. The above are some of the open issues that academics and experts that use GWO are confronted with. It is our believe that this work will encourage deliberation, and motivate upcoming research to expand our understanding of GWO, and advance the development of more effective and robust algorithms that can handle dynamic real world problem efficiently.

Another important open issue is in the area of population diversity. This can be described as is the magnitude of population status with respect to exploration or exploitation. It demonstrates the communication of wolves' position, coefficient vector of the prey, and cognitive ability. Wolves moving in different direction indicates that the algorithm is in an exploration situation, in the reverse case, wolves congregating into a miniature search locale signifies that the algorithm in an exploitation situation. The miscellany of population of wolves is beneficial for quantifying and dynamically regulating the capacity of the algorithm for either exploration or exploitation fittingly. The wolves' positions, coefficient vector of the prey, and cognitive diversity are exploited to determine the distribution of wolves' present positions, present coefficient vector of the prey, and the best positions found thus far, correspondingly. Important information is gotten from computing the diversity. Small diversity, whereby wolves congregate into a tiny search area, is usually considered as the foremost reason for premature convergence. A number of methods have been proposed to encourage diversity in GWO [32, 36, 45, 49, 51, 53, 62-66]. However, more research has to be done in this area to overcome the problem of wolves converging too early in the search space.

Also, more work have need to be done to get more understanding and optima tuning of parameters to obtain better results for GWO algorithms. In Softcomputing research, one of the main thing that reviewers usually emphasize for a novel algorithm is giving an unbiased comparison for all the algorithms in the work. The reason is to ensure that authors have meticulously done their parameter tuning of values so that the algorithms under consideration can produce the most ideal results as much as possible on their benchmark functions. However, it is an open secret that many researcher did not tune the parameter values for the other techniques they compared with their proposed method. Despite the recent advances in GWO algorithms, there is no standard definition for what an unbiased comparison among all algorithms means. Usually, the 
proposed algorithm and the other ones been compared are tested on a set of new benchmark functions, which are not the same as the benchmark functions employed by other algorithms as at the time they were initially presented. It is still an open research problem whether every algorithms must have identical parameter settings or not. Moreover, knowing whether individual algorithm has precisely very similar settings compared to the settings when it was initially proposed or maybe they should all have the similar configuration variable settings. In summary, the open research problems in GWO are enumerated below:

- The issue of parameter tuning of GWO algorithms need to be properly investigated. It is a common knowledge that only two parameters are used to maintain balance between exploration and exploitation during GWO search, and the values are predetermined and left unchanged throughout the search. This can pose a serious challenge to inexperienced users with little knowledge about the workings of GWO algorithms [76]. The feasibility of having varied number of wolves in each iteration during search operations need to be researched.

- Nonexistence of effective approach to adapt or use the GWO algorithm to proffer solution to dynamic problems that requires changes in global optimum over time. Though the MOGWO variant has proven to have great exploration capacity in detecting distinctive areas of a search space, it however requires adjustment to update the solutions that offer apt concession between all objectives without any form of deterioration.

- Unavailability of research work that focused on tuning the parameters of GWO algorithm for solving practical problems.

- The possibility of fusing GWO with other conventional optimisation techniques like prima dual interior point method, steepest descent method, conjugate gradient method, quasi-Newton technique, Taguchi technique, linear programming, integer programming, or dynamic programming for robust optimisation is another open research area that is waiting to be explored.

- Absence of research work on how best to use GWO algorithm to solve constrained optimization problems. Notwithstanding the recent progress that have been achieved in using GWO to solve practical world problems, there is a dearth research work that study the most ideal approach to handle constraints for GWO. Nothing has been done to develop variants of GWO that possess more adaptable and application-oriented constraint schemes. A greater part of existing work in literature employed static penalty constraints handling method, and this has proven to be unproductive in situations where the search space is characterised with many area that have no solution which satisfies all the constraints. We hereby recommend that researchers should concentrate on developing GWO variants that have the capacity to effectively handle constraints in several ways.

- There is need to develop more robust GWO variants that support locating dependable and consistent solutions. This is very essential for proffering solution to practical problems that are characterized with numerous doubts relating to inputs, outputs, functions to minimize or maximize in the problem, and limits. No research has been conducted to find out how such disruptions influence the ability of GWO to find effective and stable solutions.

- Some progress has been made in the past on the use GWO to solve binary problems, for instance, reducing the number of input variables when developing a predictive model. Nevertheless, there is no existence of a standardized generic binary model for GWO. Feature selection problem are continuous in nature and therefore require binary version of GWO. For the algorithm to be able to provide solution for a variety of binary problems, there is need to incorporate another transfer functions (s-shaped and v-shaped). This will assist in updating the features of binary solution from 0 to 1 and contrariwise [77].

- Despite the existence of MOGWO algorithm, a number of research gaps need to be bridged. This will enable GWO to be able to compete favourably with other Softcomputing algorithms in solving multi-objective problems in a cost effective manner. MOGWO employs an archive for its operations. However, apart from archive, other approaches such as applying varied operators such as niching, aggregation technique, nondominated sorting, efficient nondominated sort and others need to be explored to provide computationally cheap solutions to multi-objective optimization problems. 
- The existing multi-objective variants of GWO utilize Pareto efficiency dominance to provide contrast among solutions. In continuation of the above discussion, an archive keeps solutions that gives a proper concession among every objectives without allowing any one of them to deteriorate. When a problem has up to four objectives, they are moderately handled by archive in an effective manner. On the other hand, archive has proven to be computationally expensive and inefficient when handling many-objective problems. The reason for this is that it gets full very fast because to the abundance of feasible solutions that does not worse the other objective function. It is therefore necessary to incorporate some distinctive operators to solve many multi-objective problems. Incorporating operators such as hypervolume, near Pareto optimal dominance, modified Pareto optimal dominance, and niching will surely enhance the performance of GWO algorithms.

- Deficiency of publications that did indepth hypothetical study on GWO optimization structure is another open research area. Such study will result into researchers having additional deep and concrete knowledge of the existing GWO variants and robust response to the question of what is responsible for GWO superior performance over other state-of-the-art algorithms. Consequently, it is important to do a thorough and all-inclusive hypothetical investigation of the key characteristics of GWO algorithm. The qualities that need to be investigated comprise configuration of the wolves' population, parameters, and adaptive terrains. There is need to research into the reason for having only three leaders (alpha wolves) in the GWO algorithm, what effect it will have on the performance if the number of alpha wolves is changed either by adding or reducing it.

\section{CASE STUDIES OF APPLICATIONS OF GWO ALGORITHM FROM LITERATURE}

The Binary GWO algorithm was hybridized with Fuzzy k-Nearest Network (BGWOA-FKNN) and used for email spam filtering in [78]. Email spam is a major problem that has caused many financial loss to institutions and individuals who have fallen victim to fraudulent activities of spammers [79]. The authors used SPAMBASE dataset for their experiment. The performance of the proposed model was compared with other models such as Random committee, Naïve Bayes, Rotation Forest, Random Forest, hybrid of Binary Whales Optimisation Algorithm and Fuzzy k-Nearest Network. Experimental results shows that BGWOA-FKNN with accuracy (97.61\%), Precision (100), Sensitivity (96.27), and Specificity (100) performed better than all the other models considered in the work. This proved the efficacy of GWO algorithm.

The authors in [80] proposed a novel model that fuses an improved grey wolf optimization (IGWO), and kernel extreme learning machine (KELM) for diagnosing medical ailments. The model is called IGWOKELM. Simulation results shows that IGWO-KELM approach have the best performance in terms of the amount of chosen features, accuracy, sensitivity, specificity, precision, G-mean, and F- measure. This work is another testimony of the superiority of GWO algorithm over many other existing bio-inspired algorithms.

\section{CONCLUSION AND FUTURE RESEARCH}

This study presents a review of applications of GWO in several fields to provide researchers and engineers with a simple to comprehend applications of GWO. This will motivate researchers to propose new variants of GWO that addresses the deficiencies of the existing versions of the algorithm. Grey wolf Optimisation can be basically categorised as the single-objective GWO and the multi-objective GWO. The former is devoted to providing solutions to single objective problems, while the latter is for solving multiple objective problems. This review showed that GWO algorithms have drawn attention from several quarters and the algorithms are being applied in areas such as: classical engineering design problems, image processing, clustering, surface waves, job scheduling, Unmanned Combat Ariel Vehicle (UCAV), Optimal Reactive Power Dispatch (ORPD), Bankruptcy Prediction, Economic Load Dispatch Problem and Smart Green House. Results suggestions from the applications of the GWO indicated that the GWO is an improvement on the performance of existing bio-inspired algorithms like BA, GA, FA, PSO, and others. It is obvious from this study that the applications of GWO are few in the literature because of the newness of the algorithm and is presently being understudied by the research community. 
However, despite its newness, application of GWO increases over the years as depicted in Figure 8. Though some hybridized variants of GWO have been proposed, in the future, we intend developing our own variants of GWO by hybridizing it with other algorithms such as MFO, Harris Hawks Optimization (HHO), League Championship Algorithm (LCA), Firefly algorithm (FA) and Dragonfly algorithm (DA). This will help in producing GWO variants with better results. Also, the BGWO approach proposed in [37] was only tested for a single objective function, the same can be used to solve multi-objective problems in other domains. Furthermore, there is a need to test most of the proposed variants of GWO algorithm with large real datasets. In conclusion, understanding of the most suitable parameter values is needed for the implementation of GWO to solve problems in any field, and any bio-inspired algorithm can be integrated into GWO to improve the performance of GWO significantly. GWO can further be explored in other domains such as swarm robotic, network traffic management, quantum computing, crude oil production, big data, data mining, image segmentation, cloud computing, email spam filtering, and others. Finally, grey wolf optimization algorithm is promising for solving optimisation problems in several fields of studies.

\section{CONFLICTS OF INTEREST}

No conflict of interest was declared by the authors.

\section{REFERENCES}

[1] Rezaei, H., Bozorg-Haddad, O., Chu, X., "Grey wolf optimization (GWO) algorithm", In Advanced Optimization by Nature-Inspired Algorithms, Springer, Singapore, 81-91, (2018).

[2] Kennedy, J., Eberhart, R., "Particle swarm optimization", In Proceedings of ICNN'95International Conference on Neural Networks, IEEE, 4: 1942-1948, (1995).

[3] Karaboga, D., Basturk, B., "A powerful and efficient algorithm for numerical function optimization: artificial bee colony (ABC) algorithm”, Journal of Global Optimization, 39(3): 459471, (2007).

[4] Yang, X. S., “A new metaheuristic bat-inspired algorithm”, In: Juan R. González, David Alejandro Pelta, Carlos Cruz, Germán Terrazas, Natalio Krasnogor, Nature Inspired Cooperative Strategies for Optimization, 284, 65-74, (2010).

[5] Pham, D. T., Ghanbarzadeh, A., Koç, E., Otri, S., Rahim, S., Zaidi, M., "The bees algorithm - a novel tool for complex optimisation problems", In Intelligent production machines and systems, Elsevier Science Ltd, 454-459, (2006).

[6] Mucherino, A., Seref, O., "Monkey search: a novel metaheuristic search for global optimization", In AIP conference proceedings, American Institute of Physics, 953(1): 162-173, (2007).

[7] Krishnanand, K. N., Ghose, D., "Detection of multiple source locations using a glowworm metaphor with applications to collective robotics", In Proceedings 2005 IEEE Swarm Intelligence Symposium, SIS 2005, IEEE, 84-91, (2005).

[8] Passino, K. M., "Biomimicry of bacterial foraging for distributed optimization and control", Control Systems, IEEE, 3, 52-67, (2002).

[9] Li, X. L., "An optimizing method based on autonomous animats: fish-swarm algorithm", Systems Engineering-Theory and Practice, 22(11): 32-38, (2002). 
[10] Chu, S. A., Tsai, P. W., Pan, J. S., "Cat swarm optimization”, Lecture Notes in Computer Science (including subseries Lecture Notes in Artificial Intelligence and Lecture Notes in Bioinformatics), 4099: LNAI: 854-858, (2006).

[11] Fister, Jr. I., Yang, X. S., Fister, I., Brest, J., Fister, D., "A brief review of nature-inspired algorithms for optimization", arXiv preprint arXiv: 1307.4186, (2013).

[12] Yang, X. S., "Flower pollination algorithm for global optimization", In International conference on unconventional computing and natural computation, Springer, Berlin, Heidelberg, 240-249, (2012).

[13] Meng, X., Liu, Y., Gao, X., Zhang, H., "A new bio-inspired algorithm: chicken swarm optimization", In International conference in swarm intelligence, Springer, Cham, 86-94, (2014).

[14] Jiang, H., Zhang, S., Ren, Z., Lai, X., Piao, Y., “Approximate muscle guided beam search for three-index assignment problem", In International Conference in Swarm Intelligence, Springer, Cham., 44-52, (2014).

[15] Mo, H., Liu, L., Geng, M., "A magnetotactic bacteria algorithm based on power spectrum for optimization", In International Conference in Swarm Intelligence, Springer, Cham, 115-125, (2014).

[16] Wang, G. G., Deb, S., Coelho, L. D., "Elephant herding optimization”, In 2015 3rd International Symposium on Computational and Business Intelligence (ISCBI) 2015 Dec 7, IEEE, 1-5, (2015).

[17] Findik, O., "Bull optimization algorithm based on genetic operators for continuous optimization problems", Turkish Journal of Electrical Engineering and Computer Sciences, 23 (Sup. 1): 222539, (2015). Doi: 10.3906/elk-1307-123

[18] Mirjalili, S., Mirjalili, S. M., Lewis, A., "Grey wolf optimizer", Advances in Engineering Software, 69, 46-61, (2014).

[19] Gholizadeh, S., "Optimal design of double layer grids considering nonlinear behaviour by sequential grey wolf algorithm". Iran University of Science and Technology, 5(4), 511-523, (2015).

[20] Mirjalili, S., "How effective is the Grey Wolf optimizer in training multi-layer perceptrons", Applied Intelligence, 43(1), 150-161, (2015).

[21] Saremi, S., Mirjalili, S. Z., Mirjalili, S. M., "Evolutionary population dynamics and grey wolf optimizer", Neural Computing and Applications, 26(5), 1257-1263, (2015).

[22] Sulaiman, M. H., Mustaffa, Z., Mohamed, M. R., Aliman, O., "Using the gray wolf optimizer for solving optimal reactive power dispatch problem", Applied Soft Computing, 32, 286-292, (2015).

[23] El-Fergany, A. A., Hasanien, H. M., "Single and multi-objective optimal power flow using grey wolf optimizer and differential evolution algorithms", Electric Power Components and Systems, 43(13), 1548-1559, (2015).

[24] Madadi, A., Motlagh, M. M., "Optimal control of DC motor using grey wolf optimizer algorithm", Technical Journal of Engineering and Applied Science, 4(4), 373-379, (2014).

[25] Guha, D., Roy, P. K., Banerjee, S., "Load frequency control of interconnected power system using grey wolf optimization", Swarm and Evolutionary Computation, 27, 97-115, (2016). 
[26] Song, X., Tang, L., Zhao, S., Zhang, X., Li, L., Huang, J., Cai, W., "Grey wolf optimizer for parameter estimation in surface waves", Soil Dynamics and Earthquake Engineering, 75: 147-157, (2015). DOI: http://dx.doi.org/10.1016/j.soildyn.2015.04.004

[27] Faris, H., Aljarah, I., Al-Betar, M. A., Mirjalili, S., "Grey wolf optimizer: a review of recent variants and applications", Neural Computing and Applications. 30(2): 413-35, (2018).

[28] Hatta, N. M., Zain, A. M., Sallehuddin, R., Shayfull, Z., Yusoff, Y., "Recent studies on optimisation method of Grey Wolf Optimiser (GWO): a review (2014-2017)", Artificial Intelligence Review, 52(4): 2651-2683, (2018).

[29] Panda, M., Das, B. "Grey Wolf Optimizer and Its Applications: A Survey”, In Proceedings of the Third International Conference on Microelectronics, Computing and Communication Systems, Springer, Singapore, 179-194, (2019). DOI: https://doi.org/10.1007/978-981-13-7091-5_17

[30] Al-Tashi, Q., Rais, H. M., Abdulkadir, S. J., Mirjalili, S., Alhussian, H., "A Review of Grey Wolf Optimizer-Based Feature Selection Methods for Classification", In Evolutionary Machine Learning Techniques, Springer, Singapore, 273-286, (2020). DOI: https://doi.org/10.1007/978981-32-9990-0_13

[31] Negi, G., Kumar, A., Pant, S., Ram, M., "GWO: a review and applications”, International Journal of System Assurance Engineering and Management, 1-8, (2020). DOI: https://doi.org/10.1007/s13198-020-00995-8

[32] Yang, B., Zhang, X., Yu, T., Shu, H., Fang, Z., "Grouped grey wolf optimizer for maximum power point tracking of doubly-fed induction generator based wind turbine", Energy Conversion and Management, 133: 427-43, (2017). DOI: http://dx.doi.org/10.1016/j.enconman.2016.10.062

[33] Lal, D. K., Barisal, A. K., Tripathy, M., "Grey wolf optimizer algorithm based Fuzzy PID controller for AGC of multi-area power system with TCPS", Procedia Computer Science, 92: 99105, (2016). DOI: 10.1016/j.procs.2016.07.329

[34] Precup, R. E., David, R. C., Petriu, E. M., Szedlak-Stinean, A. I., Bojan-Dragos, C. A., "Grey wolf optimizer-based approach to the tuning of pi-fuzzy controllers with a reduced process parametric sensitivity”, IFAC - PapersOnLine, 49(5): 55-60, (2016). DOI: 10.1016/j.ifacol.2016.07.089

[35] Debnath, M. K., Mallick, R. K., Sahu, B. K., "Application of hybrid differential evolution-grey wolf optimization algorithm for automatic generation control of a multi-source interconnected power system using optimal fuzzy-PID controller", Electric Power Components and Systems, 45(19): 2104-17, (2017). DOI: 10.1080/15325008.2017.1402221

[36] Lu, C., Xiao, S., Li, X., Gao, L., "An effective multi-objective discrete grey wolf optimizer for a real-world scheduling problem in welding production", Advances in Engineering Software, 99, 161-176, (2016). DOI: http://dx.doi.org/10.1016/j.advengsoft.2016.06.004

[37] Panwar, L. K., Reddy, S., Verma, A., Panigrahi, B. K., Kumar, R., "Binary grey wolf optimizer for large scale unit commitment problem", Swarm and Evolutionary Computation, 38: 251-66, (2018). DOI: http://dx.doi.org/10.1016/j.swevo.2017.08.002

[38] Komaki, G. M., Kayvanfar, V., "Grey Wolf Optimizer algorithm for the two-stage assembly flow shop scheduling problem with release time", Journal of Computational Science, 8: 109-20, (2015). DOI: http://dx.doi.org/10.1016/j.jocs.2015.03.011 
[39] Kamboj, V. K., “A novel hybrid PSO-GWO approach for unit commitment problem”, Neural Computing and Applications, 27(6): 1643-55, (2016).

[40] Ab Rashid, M. F. F., "A hybrid Ant-Wolf Algorithm to optimize assembly sequence planning problem”, Assembly Automation, (2017).

[41] Zhang, S., Zhou, Y., Li, Z., Pan, W., "Grey wolf optimizer for unmanned combat aerial vehicle path planning", Advances in Engineering Software, 99, 121-136, (2016). DOI: http://dx.doi.org/10.1016/j.advengsoft.2016.05.015

[42] Jain, U., Tiwari, R., Godfrey, W. W., "Odor source localization by concatenating particle swarm optimization and Grey Wolf optimizer", In Advanced Computational and Communication Paradigms, Springer, Singapore, 145-153, (2018).

[43] Pradhan, M., Roy, P. K., Pal, T., "Grey wolf optimization applied to economic load dispatch problems", International Journal of Electrical Power and Energy Systems, 83: 325-34, (2016). DOI: http://dx.doi.org/10.1016/j.ijepes.2016.04.034

[44] Pradhan, M., Roy, P. K., Pal, T., "Oppositional based grey wolf optimization algorithm for economic dispatch problem of power system", Ain Shams Engineering Journal, 9(4): 2015-25, (2018). DOI: http://dx.doi.org/10.1016/j.asej.2016.08.023

[45] Jayabarathi, T., Raghunathan, T., Adarsh, B. R., Suganthan, P. N., "Economic dispatch using hybrid grey wolf optimizer", Energy, 111: 630-41, (2016). DOI: http://dx.doi.org/10.1016/j.energy.2016.05.105

[46] Chopra, N., Kumar, G., Mehta, S., "Hybrid GWO-PSO algorithm for solving convex economic load dispatch problem", International Journal of Research in Advent Technology , 4(6): 37-41, (2017).

[47] Eid, H. F., Abraham, A., "Plant species identification using leaf biometrics and swarm optimization: A hybrid PSO, GWO, SVM model", International Journal of Hybrid Intelligent Systems, 14(3): 155-65, (2017).

[48] Vinothini, J., Bakkiyaraj, R. A., "Grey Wolf Optimization Algorithm for Colour Image Enhancement Considering Brightness Preservation Constraint", International Journal of Emerging Trends in Science and Technology, 03(05): 4049-4055, (2015). DOI: http://dx.doi.org/10.18535/ijetst/v3i05.28

[49] Lakshminarayanan, S., "Nature inspired grey wolf optimizer algorithm for minimizing operating cost in green smart home", Doctoral Dissertation, University of Toledo, (2015).

[50] Medjahed, S. A., Saadi, T. A., Benyettou, A., Ouali, M., "Gray wolf optimizer for hyperspectral band selection", Applied Soft Computing, 40: 178-86, (2016). DOI: http://dx.doi.org/10.1016/j.asoc.2015.09.045

[51] Mirjalili, S., Saremi, S., Mirjalili, S. M., Coelho, L. D. S., "Multi-objective grey wolf optimizer: a novel algorithm for multi-criterion optimization", Expert Systems with Applications, 47, 106119, (2016). DOI: http://dx.doi.org/10.1016/j.eswa.2015.10.039

[52] Kohli, M., Arora, S., "Chaotic grey wolf optimization algorithm for constrained optimization problems", Journal of Computational Design and Engineering, 5(4): 458-72, (2018). DOI: http://dx.doi.org/10.1016/j.jcde.2017.02.005 
[53] Singh, N., Singh, S. B., "A novel hybrid GWO-SCA approach for optimization problems", Engineering Science and Technology, an International Journal, 20(6): 1586-601, (2017). DOI: https://doi.org/10.1016/j.jestch.2017.11.001

[54] Chandra, M., Agrawal, A., Kishor, A., Niyogi, R., "Web service selection with global constraints using modified gray wolf optimizer", In: 2016 International Conference on Advances in Computing, Communications and Informatics (ICACCI), IEEE, 1989-1994, (2016).

[55] Singh, N., Singh, S. B., "Hybrid algorithm of particle swarm optimization and grey wolf optimizer for improving convergence performance", Journal of Applied Mathematics, (2017).

[56] ElGayyar, M., Emary, E., Sweilam, N. H., Abdelazeem, M., "A hybrid Grey Wolf-bat algorithm for global optimization", In International Conference on Advanced Machine Learning Technologies and Applications, Springer, Cham., 3-12, (2018).

[57] Heidari, A. A., Pahlavani, P., "An efficient modified grey wolf optimizer with Lévy flight for optimization tasks", Applied Soft Computing, 60: 115-34, (2017). DOI: http://dx.doi.org/10.1016/j.asoc.2017.06.044

[58] Jitkongchuen, D., Phaidang, P., Pongtawevirat, P., "Grey wolf optimization algorithm with invasion-based migration operation", In2016 IEEE/ACIS 15th International Conference on Computer and Information Science (ICIS), IEEE, 1-5, (2016).

[59] Pan, J. S., Dao, T. K., Chu, S. C., "A novel hybrid GWO-FPA algorithm for optimization applications", In International Conference on Smart Vehicular Technology, Transportation, Communication and Applications, Springer, Cham., 274-281, (2017).

[60] Zhang, X., Kang, Q., Cheng, J., Wang, X., "A novel hybrid algorithm based on biogeographybased optimization and grey wolf optimizer", Applied Soft Computing. 67: 197-214, (2018). DOI: https://doi.org/doi:10.1016/j.asoc.2018.02.049

[61] Tawhid, M. A., Ali, A. F., "A hybrid grey wolf optimizer and genetic algorithm for minimizing potential energy function”, Memetic Computing, 9(4): 347-59, (2017).

[62] Jeet, K., "Grey wolf algorithm for software organization", Indian Journal of Scientific Research, 7(2): 214-217, (2017).

[63] Jadhav, A. N., Gomathi, N., "WGC: hybridization of exponential grey wolf optimizer with whale optimization for data clustering", Alexandria Engineering Journal, 2018 Sep 1, 57(3): 1569-84, (2017). DOI: http://dx.doi.org/10.1016/j.aej.2017.04.013

[64] Wang, M., Chen, H., Li, H., Cai, Z., Zhao, X., Tong, C., Li, J., Xu, X., “Grey wolf optimization evolving kernel extreme learning machine: Application to bankruptcy prediction", Engineering Applications of Artificial Intelligence, 63: 54-68, (2017). DOI: http://dx.doi.org/10.1016/j.engappai.2017.05.003

[65] Emary, E., Yamany, W., Hassanien, A. E., Snasel, V., "Multi-objective gray-wolf optimization for attribute reduction", Procedia Computer Science, January 2015, 1: 65: 623-32, (2015). DOI: http://dx.doi.org/10.1016/j.neucom.2015.06.083

[66] Tu, Q., Chen, X. and Liu, X., "Multi-strategy ensemble grey wolf optimizer and its application to feature selection", Applied Soft Computing, 76, 16-30, (2019). DOI: https://doi.org/10.1016/j.asoc.2018.11.047 
[67] Hu, P., Pan, J. S., Chu, S. C., Improved Binary Grey Wolf Optimizer and Its application for feature selection. Knowledge Based Systems, 105746, (2020). DOI: https://doi.org/10.1016/j.knosys.2020.105746

[68] Li, Q., Chen, H., Huang, H., Zhao, X., Cai, Z. N., Tong, C., Liu, W., Tian, X., “An enhanced grey wolf optimization based feature selection wrapped kernel extreme learning machine for medical diagnosis", Computational and Mathematical Methods in Medicine, 2017: 15, (2017). DOI: https://doi.org/10.1155/2017/9512741

[69] Jayapriya, J., Arock, M., “A parallel gwo technique for aligning multiple molecular sequences”, In: 2015 International Conference on Advances in Computing, Communications and Informatics (ICACCI), IEEE, 210-215, (2015).

[70] Mostafa, A., Fouad, A., Houseni, M., Allam, N., Hassanien, A. E., Hefny, H., Aslanishvili, I., “A hybrid grey wolf based segmentation with statistical image for ct liver images", In: International Conference on Advanced Intelligent Systems and Informatics, Springer, 846-855, (2016).

[71] Sahoo, A., Chandra, S., "Multi-objective grey wolf optimizer for improved cervix lesion classification", Applied Soft Computing, 52: 64-80, (2017).

[72] Elhariri, E., El-Bendary, N., Hassanien, A. E., "A hybrid classification model for EMG signals using grey wolf optimizer and SVMs", In: The 1st International Conference on Advanced Intelligent System and Informatics (AISI2015), November 28-30, 2015, Beni Suef, Egypt, Springer, 297-307, (2016).

[73] Diwan, P., Khan, M. R., "Energy efficient communication for WSNs using Grey-Wolf optimization algorithm", International Journal of Engineering And Computer Science, 5(12), (2016).

[74] Editorial, “Hybrid learning machine”, Neurocomputing, 72: 2729-2730, (2009).

[75] Niu, P., Niu, S., Chang, L., "The defect of the Grey Wolf optimization algorithm and its verification method", Knowledge-Based Systems, 1: 171: 37-43, (2019).

[76] Rodriguez, L., Castillo, O., Soria, J., "A study of parameters of the grey wolf optimizer algorithm for dynamic adaptation with fuzzy logic", In: Nature-Inspired Design of Hybrid Intelligent Systems. Springer, 371-390, (2017).

[77] Mafarja, M., Eleyan, D., Abdullah, S., Mirjalili, S., "S-Shaped vs. V-Shaped Transfer Functions for Ant Lion Optimization Algorithm in Feature Selection Problem", In Proceedings of International Conference on Future Networks and Distributed Systems, Cambridge, UK, July 2017, (ICFNDS) 2017, 6, (2017). DOI: 10.1145/3102304.3102325

[78] Hassani, Z., Hajihashemi, V., Borna, K., Sahraei Dehmajnoonie, I., “A Classification Method for E-mail Spam Using a Hybrid Approach for Feature Selection Optimization”, Journal of Sciences, Islamic Republic of Iran, 31(2): 165-173, (2020).

[79] Dada, E. G., Bassi, J. S., Chiroma, H., Adetunmbi, A. O., Ajibuwa, O. E., "Machine learning for email spam filtering: review, Approaches and Open Research Problems", Heliyon, 5(6), p.e01802 (2019).

[80] Li, Q., Chen, H., Huang, H., Zhao, X., Cai, Z., Tong, C., Liu, W. and Tian, X., “An enhanced grey wolf optimization based feature selection wrapped kernel extreme learning machine for medical diagnosis", Computational and Mathematical Methods in medicine, (2017). 